\title{
Nuevos líderes, ¿nuevos discursos? Análisis en Twitter de la campaña electoral de Andrés Arauz en Ecuador (2021)
}

New leaders, new speeches? Twitter analysis of Andrés Arauz's electoral campaign in Ecuador (2021)

RESUMEN: La irrupción de las redes sociales ha venido a modificar no solo los hábitos de consumo de información, sino también los propios procesos políticos y electorales. En este marco, y atendiendo a las propias características de la información digital, los discursos políticos se han visto asimismo afectados al tener que adaptarse a los nuevos formatos. Todo ello, en un contexto de creciente personalización de la política $\mathrm{y}$, además, en un período en el que conviven liderazgos populistas con otros emergentes. En este trabajo realizamos un análisis del discurso del candidato presidencial Andrés Arauz en redes sociales (Twitter) durante la primera vuelta de la campaña electoral ecuatoriana de 2021 con el objetivo de identificar si incorpora características populistas y cuáles son sus particularidades. Para ello, se aplica un análisis del discurso al corpus formado por todos los tuits emitidos por la cuenta del candidato (@ecuarauz) durante la primera vuelta de la campaña electoral. En total, se analizan 341 tuits. Los resultados muestran que el discurso en redes de Arauz no tiene rasgos populistas y que se caracteriza por tener un tono sofisticado, construido a partir de tres campos: la esperanza, las propuestas de campaña y la crisis política.

Palabras clave: discurso, populismo, liderazgo, campaña electoral, redes sociales, Ecuador.

ABSTRACT: The emergence of social media has come to modify not only the habits of information consumption, but also the political and electoral processes 
themselves. In this framework, and taking into account the very characteristics of digital information, political speeches have also been affected by having to adapt to new formats. All this, in a context of increasing personalization of politics and, furthermore, in a period in which populist leaderships coexist with other emerging ones. In this paper we carry out an analysis of the speech of the presidential candidate Andrés Arauz on social media (Twitter) during the Ecuadorian elections of 2021with the aim of identifying if it incorporates populist characteristics and what its particularities are. To do this, we apply a discourse analysis to the corpus formed by all the tweets published by the candidate's account (@ecuarauz) during the first round of the electoral campaign. In total, 341 tweets are analyzed. The results show that Arauz discourse has no populist traits and that it is characterized by having a sophisticated tone, constructed from three fields: hope, campaign proposals and political crisis.

Key words: discourse, populism, leadership, electoral campaign, social media, Ecuador.

\section{INTRODUCCIÓN}

Las últimas décadas en América Latina han estado caracterizadas por la llegada de gobiernos progresistas identificados como populistas. Después de algunos gobiernos de transición (Macri en Argentina, Bolsonaro en Brasil, Moreno en Ecuador, Áñez en Bolivia), aparecen nuevamente líderes que retoman o continúan con los modelos de gobierno anteriores (Cristina Fernández en Argentina, Luis Arce en Bolivia). El contexto al que vuelven estos movimientos políticos es distinto a aquel en el que estos líderes o sus antecesores accedieron al gobierno, con nuevos retos, actores y estrategias políticomediáticas. Una de estas novedades es la irrupción de los medios digitales, sobre todo las redes sociales digitales, que traen consigo la posibilidad de una nueva forma de comunicación: directa, inmediata y masiva, pero también con más ruido informativo.

Este trabajo articula los estudios sobre liderazgos políticos (Laclau, 2005; Deiwiks, 2009; Alcántara at al., 2016) con aquellos que se centran en el uso de redes sociales digitales por parte de los actores políticos, sobre todo en campaña electoral (López-Meri, Marcos-García y Casero-Ripollés, 2017; García-Ortega y Zugasti-Azagra, 2018; Pérez-Curiel, Rivas de Roca y García-Gordillo, 2021) para estudiar el caso ecuatoriano y a partir de la posibilidad de que estas nuevas formas de comunicación generen discursos distintos a los de los antecesores de estos líderes. En consecuencia y como continuación de trabajos anteriores, se propone un análisis del nuevo líder del movimiento correísta: Andrés Arauz, candidato a la presidencia de Ecuador en las elecciones de 2021. En concreto, se estudia 1) si el discurso de Arauz tiene características populistas 2) cuáles son sus particularidades propias y 3) en qué medida se reflejan las características subjetivables del nuevo líder en su discurso de campaña.

\section{LOS LIDERAZGOS POPULISTAS}

En el ejercicio de representación democrática, la figura del líder y el tipo de liderazgo que ejerce resulta central, sobre todo ante los procesos de creciente personalización de la política (D’Alessadro, 2004). El liderazgo ha sido estudiado desde 
diferentes perspectivas y enfoques (D'Alessandro, 2006), lo que ha generado una variedad de conceptualizaciones en función de la dimensión desde la que se estudie. Para este trabajo, convenimos con Petracca en que los líderes son aquellos que «a) dentro de un grupo b) detentan tal posición de poder que influye en forma determinante en las decisiones de carácter estratégico, c) poder que se ejerce activamente d) y que encuentra una legitimación en su correspondencia con las expectativas del grupo» (1997: 917). A partir de las distintas propuestas, la literatura recoge un prolijo debate sobre los tipos de liderazgos políticos, que habitualmente van acompañados de una serie de adjetivos en un intento de caracterizar la manera de ejercer la política de cada actor. Aunque este trabajo no realiza un estudio sobre los tipos de liderazgos, sí conviene señalar que el tipo de liderazgo político y su calidad tienen una fuerte relación con el sistema político en el que se desempeñan, tanto con sus características como con la calidad del mismo (Alcántara et al., 2016). Por tanto, enfrentar el estudio del liderazgo político tiene no solo que ver con las características propias de cada actor, sino también con aquellas que definen al sistema al que pertenece y al contexto político en el que se desenvuelve.

Uno de los tipos de liderazgos predominante en las últimas décadas ha sido el populista. Si bien abordar el populismo no es tarea sencilla y las perspectivas que encontramos son distintas, hay algunos elementos comunes en todas las que abordan este fenómeno. El primero de ellos es la importancia dada a la categoría pueblo, sobre el cual existen asimismo diferentes posturas; el segundo es la construcción de un antagonismo entre el pueblo y los otros (Deiwiks, 2009), es decir, entre un nosotros y un ellos que marca diferentes maneras de entender la política.

Para la emergencia de los populismos, algunos autores proponen la necesidad de unas condiciones previas: la existencia de una crisis o la desconfianza en el sistema político-democracia representativa (Laclau, 2005; Deiwiks, 2009), que tendrían además como punto de partida la dicotomización del espacio social (Laclau, 2005). En el caso de América Latina, el resurgimiento de este tipo de gobierno en las últimas décadas dio paso a la configuración de un bloque antihegemónico frente a los países del Norte en materia económica y política (De la Torre, 2013). Estos liderazgos vendrían a ser el resultado de: un discurso basado en la lucha entre el pueblo y la oligarquía como una disputa entre el bien y el mal; un líder redentor; movilización social desde la élite y la incorporación de sectores tradicionalmente excluidos de la política (De la Torre, 2012: 252). A estas características tradicionales habría que añadir las habilidades y destrezas comunicativas del líder (Chavero, 2017), que facilitan la comunicación y el contacto directo entre el líder y los ciudadanos, lo que a su vez permite una mejor identificación de la ciudadanía con el líder.

En las últimas décadas, la creciente importancia de los medios de comunicación para el ejercicio de estos liderazgos populistas ha llevado a algunos autores a hablar de neo-populismo (Bouza, 2007; Hermet, 2003) o de populismo mediático (Ortega, 2011). No en vano, algunas propuestas acercan el populismo más a la comunicación y al discurso que al ejercicio político (Laclau, 2005; Jagers \& Walgrave, 2007; Carsten Reinemann et al., 2016), destacando con ello la importancia del discurso para identificar la presencia y el grado de populismo en los líderes políticos. Por tanto, el análisis de los tipos de liderazgos, en especial los populistas, nos lleva al campo del discurso, como el espacio y producto donde confluyen los elementos que caracterizan al líder y las estrategias (o una parte de ellas) que emplea. Si bien entendemos el discurso como una práctica social (Charaudeau, 2009) en la que convergen las condiciones sociales, económicas y políticas, en este trabajo partimos del discurso en tanto se relaciona con la ideología y la política, dado que los discursos políticos producen y reproducen las prácticas y las ideologías políticas (Van Dijk, 2005) y permiten identificar las relaciones de poder (Van Dijk, 2009). 


\section{LA CAMPAÑA ELECTORAL Y LAS REDES SOCIALES}

Dentro del ejercicio político, los procesos electorales son uno de los momentos de mayor interés, por ser el período en el que los líderes se muestran más cercanos a la ciudadanía (Paletz, 1997). Las campañas electorales son el momento en el que se disputa el poder, por lo que el ejercicio político incrementa su intensidad a través de la puesta en marcha de estrategias específicas de negociación y ataque entre quienes participan de la contienda. La abundante literatura académica sobre estos procesos se divide en grandes áreas: desde sus efectos en la ciudadanía y en la decisión de voto (Canel, 1998, Muñoz Alonso y Rospir, 1995; García Beadoux y D’Adamo, 2004; Martínez i Coma, 2008) hasta el desarrollo de la propia campaña o la manera en que los medios las cubren (Gitlin, 1991; De Vreese, 2004). Dentro de esta última línea de estudio, los medios de comunicación han ido tomando cada vez más protagonismo, no solo durante los períodos electorales, sino durante el ejercicio político en general. Una de las consecuencias de este rol central de los medios es la adaptación de los actores políticos a los procesos mediáticos, en particular en lo relativo al discurso, lenguaje y acciones, pero también en lo referido a la toma de decisiones (Mazzoleni y Schutz, 1999; Vallés, 2010). En un paso más de este recorrido, en la última década asistimos a nuevos cambios producidos por la irrupción de las herramientas digitales, en especial de las redes sociales (Yang et. Al, 2016).

La generalización del consumo digital ha traído consigo la creación de una esfera virtual, que tiene características con las que se puede mejorar la democracia (mayor participación), pero que no está exenta de desigualdades y múltiples esferas en su interior (Papacharissi, 2002). Esta nueva virtualidad está teniendo impactos en distintos sectores de la vida pública: la Administración Pública, los movimientos sociales y la propia actividad política, en especial en lo relativo a los líderes, desde su discurso hasta la forma de interactuar con otros líderes y la ciudadanía e incluso la manera de elaborar la política pública.

La red, caracterizada por la multilateralidad, la interacción, la gratuidad, la inmediatez y la mayor capacidad de difusión, permite un mayor consumo y replicabilidad de la información (Cotarelo, 2012), lo que aumenta exponencialmente el tráfico de datos entre los usuarios, si bien la permanencia de una brecha digital sigue impidiendo que la información llegue a todos los sectores sociales. Dentro de esta virtualidad, las redes sociales están ocupando un lugar protagonístico en la vida pública, tanto por sus potencialidades (interacción, participación, mayor consumo) como por los riesgos (fake news, desinformación) que conlleva para la democracia e incluso algunas propuestas hablan de un pseudodebate en la red (Chavero, 2013). Una de las líneas de estudio que más está creciendo es la relativa al uso que hacen los líderes políticos de las redes durante los procesos electorales, cuyos trabajos dan cuenta de que la función predominante es la de difusión de las propuestas, al tiempo que se da una creciente hibridación entre las redes y los medios convencionales (López-Meri, Marcos-García y Casero-Ripollés, 2017; García-Ortega y Zugasti-Azagra, 2018), lo que genera una creciente autorreferencialidad de los líderes políticos en Twitter (García-Ortega y Zugasti-Azagra, 2018). Algunos autores alertan de que esta creciente hibridación es también un indicador de un gran número de operaciones de fake news mills -generador de noticias falsas- que tienen un especial protagonismo durante las campañas electorales (Calvo y Aruguete, 2020). Otros trabajos se centran en el uso de la imagen y la fotografía a la hora de construir los liderazgos políticos en redes, sobre todo por la posibilidad de acercarse al ciudadano (López-Rabadán, López-Meri y Doménech-Fabregat, 2016). 
El uso político de las redes sociales tiene recorridos diferentes en función del tipo de liderazgo. Por un lado, los dirigentes populistas de izquierda son más propensos a tratar temas relacionados con los problemas sociales, corrupción y regeneración democrática (Alonso-Muñoz y Casero-Ripollés, 2018). Por otro lado, los líderes populistas de extrema derecha emplean recursos retóricos y estratégicos como mecanismos de propaganda, con la utilización de la emoción, un lenguaje sencillo y ataques a los adversarios (PérezCuriel, Rivas de Roca y García-Gordillo, 2021). Como parte de esta estrategia, los líderes populistas apuestan en Twitter por un discurso ideológico en torno a una agenda homogénea (migración, terrorismo o relaciones exteriores) que se combina con información de naturaleza más personal (Pérez Curiel, 2020).

\section{EL CASO DE ECUADOR: LAS ELECCIONES DE 2021}

El sistema político ecuatoriano se ha caracterizado tradicionalmente por una fuerte inestabilidad y fragmentación, hasta que la llegada al gobierno de Rafael Correa (20072017) dio paso al partido hegemónico (Freidenberg y Pachano, 2016). Esta hegemonía vuelve a cambiar después de 2017, cuando asume la presidencia, de la mano del propio Correa, Lenín Moreno. El nuevo presidente rompió no solo con el tipo de liderazgo de Correa, sino con el proyecto político que le había llevado al poder y pronto se alineó con las élites, los grandes poderes económicos y la que hasta entonces había sido oposición política. De esta manera, durante los cuatro años de su gobierno implementó la agenda neoliberal que había perdido la elecciones en 2017 y volvió a tejer alianzas con los propietarios de los medios de comunicación y del sector financiero, incluido el Fondo Monetario Internacional, que durante el gobierno de Correa habían sido adversarios políticos (Arias y Chiriboga, 2020). Este divorcio supuso la fractura y el debilitamiento del movimiento político Alianza País, que le había llevado al gobierno y que prácticamente desaparece tras las elecciones de 2021, no sin antes expulsar al propio Moreno por incumplimiento del programa de gobierno.

La ruptura de Lenín Moreno con el proyecto político se evidenció con la consulta popular de 2018, con la que se nombra a un Consejo de Participación Ciudadana y Control Social (CPCCS, órgano de participación ciudadana creado por el gobierno de Correa) de carácter transitorio para designar a nuevas autoridades de control nacional (Consejo Nacional Electoral, Consejo de la Judicatura, Corte Constitucional, Tribunal Contencioso Electoral o Fiscal General del Estado, entre otros) en sustitución de las elegidas por concursos púbicos de mérito y oposición. De esta manera, y de la mano del ya fallecido Julio César Trujillo -nombrado presidente del CPCCS-T a sus 87 años-, Moreno modificó toda la institucionalidad de Ecuador en lo que algunos autores han denominado deriva neofascista (Guamán, 2020).

El segundo momento importante durante el gobierno de Moreno es la crisis política de octubre de 2019. En esa fecha, Moreno aprueba una serie de medidas con las que elimina el subsidio a la gasolina ${ }^{1}$ y reduce fuertemente el Estado, afectando directamente a los trabajadores del sector público, al tiempo que reduce la presión físcal sobre las grandes fortunas. Consecuencia de esta medida, amplias capas de la población lideradas por el movimiento indígena protagonizan protestas durante 12 días por todo el país, a lo que Moreno responde militarizando Quito y cambiando la capital de ciudad temporalmente. Después de 12 días de paralización total del país y varios muertos, Moreno acepta sentarse en una mesa de negociación con representantes del movimiento

\footnotetext{
${ }^{1}$ Conviene recordar que la exportación de petróleo es la principal fuente de ingresos de la economía ecuatoriana.
} 
indígena para buscar una alternativa. Tras el conocido como paro de octubre de 2019, en el que se registraron graves violaciones de derechos humanos ${ }^{2}$, el gobierno de Moreno perdió casi toda su legitimidad (las encuestas publicadas situaban su legitimidad entre el $14 \%$ y el $8 \%$ en enero de 2020) y enfrentaba su último año de gobierno con una fuerte crisis económica, política y social. Después de cuatro años de despidos masivos, sobre todo vinculados al sector público, en diciembre de 2020 solo el 30,8\% de la población activa contaba con empleo adecuado, según las cifras oficiales ${ }^{3}$. En diciembre de 2020, la pobreza se situaba en el $32,4 \%$ de la población, en tanto que la pobreza extrema estaba en el $9 \%$, según los mismos datos ${ }^{4}$.

La pandemia generada por el covid-19 en 2020 profundizó esta triple crisis y añadió la sanitaria, en especial tras la dimisión de la ministra de Salud y el ministro de Trabajo durante los primeros días de la pandemia ${ }^{5}$, pero también con los sucesivos casos de corrupción denunciados durante los meses de pandemia y las consecuencias del debilitamiento del sistema público de salud en el gobierno de Moreno.

Junto con la pandemia, el segundo semestre de 2020 estuvo marcado por la puesta en marcha del proceso electoral para los cargos de Presidente y Vicepresidente de la República (binomio) y asambleístas. El nuevo órgano electoral (CNE), cuyos integrantes habían sido designados en 2018 cumpliendo cuotas políticas y sin representación del correísmo (primera fuerza política), protagonizó distintos intentos de impedir primero la inscripción de un nuevo partido político que acogiese a líderes correístas $\mathrm{y}$, después, impidiendo la inscripción del propio Correa como candidato a vicepresidente. Finalmente, el binomio es configurado por Andrés Arauz (presidente) y Carlos Rabascall (vicepresidente) e inscrito como candidatura de una alianza formada por Centro Democrático y Fuerza Compromiso Social, dos partidos que ya habían sido autorizados con anterioridad por el CNE. E1 8 de diciembre de 2020 son autorizados 16 binomios para las elecciones del 7 de febrero de $2021^{6}$.

Además de la posibilidad de fraude, la contienda electoral estuvo marcada por la pandemia del covid-19, que impidió la celebración de evento masivos y estableció otras limitaciones en cumplimiento de las normas de bioseguridad. En consecuencia, la campaña se desarrolla fuertemente en la esfera digital, en consonancia además con el incremento del uso de internet y las redes sociales en campaña (Cárdenas, Ballesteros y Jara, 2017).

Los resultados electorales del 7 de febrero no fueron reconocidos hasta el 21 de febrero por parte del $\mathrm{CNE}^{7}$, tras sucesivas denuncias de fraude y procesos de solicitud de recuento de votos por parte de Yaku Pérez, candidato del movimiento indígena Pachakutik. Finalmente, Andrés Arauz obtiene un 32,72\% de votos, Guillermo Lasso un $19,74 \%$ y Yaku Pérez un $19,39 \%^{8}$, con lo que Arauz (progresista) y Lasso (conservador

\footnotetext{
2 https://www.dpe.gob.ec/comision-especial-para-la-verdad-y-la-justicia-entregara-el-informe-de-loshechos-ocurridos-en-las-protestas-de-octubre-de-2019/ y https://www.oas.org/es/cidh/prensa/comunicados/2020/008.asp

${ }^{3} \mathrm{https}: / /$ www.ecuadorencifras.gob.ec/documentos/web-inec/EMPLEO/2020/Diciembre2020/202012_Mercado_Laboral.pdf

${ }^{4} \mathrm{https} / / /$ www.ecuadorencifras.gob.ec/documentos/web-inec/POBREZA/2020/Diciembre2020/202012_PobrezayDesigualdad.pdf

${ }^{5}$ Durante la gestión de Moreno de la pandemia hubo 4 ministros de Salud.

${ }^{6} \mathrm{https}: / /$ www.primicias.ec/noticias/politica/binomio-arauz-rabascall-cne-decision/

${ }^{7}$ https://Twitter.com/cnegobec/status/1363393686743240706?ref_src=twsrc\%5Etfw\%7Ctwcamp\%5Etwe etembed\%7Ctwterm\%5E1363393686743240706\%7Ctwgr\%5E\%7Ctwcon\%5Es1_\&ref_url=https\%3A\% 2F\%2Fwww.primicias.ec\%2Fnoticias\%2Fpolitica\%2Fcne-reclamo-pachakutik-recuento\%2F

${ }^{8}$ https://resultados2021.cne.gob.ec/
} 
y banquero conocido por su relación con la crisis financiera ecuatoriana de 1999) disputan la segunda vuelta electoral.

\section{PREGUNTAS DE INVESTIGACIÓN Y ESTRATEGIA METODOLÓGICA}

En este trabajo realizamos un análisis de las características del discurso de Andrés Arauz como candidato del movimiento correísta. Para ello, partimos de la caracterización de Arauz como un nuevo líder político. En lo que respecta a las características objetivables (Alcántara et al., 2016), esto es, al perfil sociodemográfico, carrera política y su trayectoria en el partido, encontramos que cuenta con una formación académica similar a la de Correa ( $\mathrm{PhD}$ (c) en Economía, con formación estadounidense), es joven (Correa alcanzó la presidencia con 44 años, Arauz es candidato con 36) y de familia no acomodada. Arauz tiene cierta experiencia en el sector público (director general del Banco Central de Ecuador entre 2009 y 2011 y ministro coordinador de Conocimiento y Talento Humano entre 2015 y 2017) y un breve recorrido en el movimiento político Alianza País, como parte de su trabajo en la Revolución Ciudadana liderada por Correa. En lo relacionado con las características subjetivables (Ibíd.), es decir, en sus habilidades y atributos, Arauz es un líder técnico y poco emocional. Todo lo antedicho nos lleva a plantear la primera pregunta de investigación:

PI.1. ¿Cuáles son los elementos populistas que incorpora el candidato correísta Arauz en su discurso durante la campaña electoral?

A partir de la literatura y atendiendo a que Arauz es el candidato designado por el propio Correa, nuestra primera hipótesis se plantea en los siguientes términos:

H1: Andrés Arauz reproduce algunas características populistas de su predecesor en su discurso electoral, tales como la apelación al pueblo, a la patria y la confrontación con los adversarios.

En segundo lugar, nos proponemos profundizar en las particularidades del nuevo líder, lo cual hacemos a partir de las siguientes interrogantes:

PI.2. ¿Cuáles son los campos semánticos y particularidades que construyen el discurso de Arauz en redes sociales digitales?

PI.3. ¿Cómo se reflejan las características subjetivables de Arauz en el lenguaje que utiliza en Twitter durante la campaña electoral?

Para guiar el análisis, planteamos que:

H2: El discurso electoral en redes de Arauz gira en torno a las propuestas de campaña y la necesidad de regenerar la vida política ecuatoriana.

H3: Las características subjetivables de Arauz permean en su discurso, por lo que utiliza un lenguaje técnico y de tono elevado, aunque incorpora elementos que lo acercan a la población durante la campaña.

Para responder a estas preguntas realizamos un análisis crítico del discurso (Van Dijk, 1999; Charaudeau, 2009), entendido como la propuesta teórico-metodológica que permite identificar las relaciones de poder y su articulación con el contexto social y político. De esta manera, el análisis del discurso permite no sólo identificar las 
condiciones discursivas en las que se emite un texto, sino también su relación con las circunstancias políticas y sociales (Fairclough, 1995). Para su operacionalización, diseñamos una matriz de análisis (Tabla 1) con variables que permiten identificar: 1) posibles características populistas; 2) principales campos semánticos en torno a los que se articula el discurso y 3) tipo de lenguaje y tono que utiliza.

El corpus de análisis está formado por todos los tuits y retuits publicados por la cuenta de Twitter del candidato Arauz (@ecuarauz) durante la campaña electoral, siendo este el principal canal oficial digital. En concreto, seleccionamos el período comprendido entre el 31 de diciembre de 2020 (inicio de la campaña electoral) y el 24 de febrero de 2021 (tres días después de la fecha en la que el CNE proclama los resultados oficiales; incluir estos días posteriores permite recoger las reacciones a la proclamación de resultados, sobre todo por las dudas generadas en torno a la gestión del CNE). En total, el corpus de análisis es de 341 tuits. Partimos de los tuits como unidades lingüísticas, pero las analizamos a la luz del contexto económico y político de la campaña y de la propia carrera política de Arauz, así como de los actores que aparecen mencionados o referenciados. Entre ellos incluimos aquellos cuyos tuits son retuiteados por la cuenta oficial del candidato, a fin de identificar los niveles de autorreferencialidad. 
Tabla 1. Categorías teóricas y variables de análisis

\begin{tabular}{|c|c|c|}
\hline Categoría & Variables & Descripción \\
\hline \multirow[t]{4}{*}{ Populismo } & Pueblo & $\begin{array}{l}\text { Utilización del pueblo como parte } \\
\text { central del discurso, apelación a él } \\
\text { y sus características. }\end{array}$ \\
\hline & Confrontación & $\begin{array}{l}\text { Ataque o relación de } \\
\text { conflictividad con otros actores, } \\
\text { construcción de un escenario } \\
\text { polarizado. }\end{array}$ \\
\hline & Élite & $\begin{array}{l}\begin{array}{l}\text { Apelación a la élite } \\
\text { composición. }\end{array} \\
\text { l su } \\
\end{array}$ \\
\hline & Patria & $\begin{array}{l}\text { Apelación o utilización de la } \\
\text { patria como un actor del mapa } \\
\text { político y su significado. }\end{array}$ \\
\hline \multirow[t]{4}{*}{ Construcción del discurso } & Lenguaje & $\begin{array}{l}\text { Tipo de lenguaje: técnico, } \\
\text { coloquial, literario, etc. }\end{array}$ \\
\hline & Campos semánticos & $\begin{array}{l}\text { Conjuntos } \\
\begin{array}{l}\text { significados } \\
\text { utilizados. }\end{array}\end{array}$ \\
\hline & Categorías lingüísticas & $\begin{array}{l}\text { Herramientas utilizadas para la } \\
\text { definición del contexto } \\
\text { (comunicativo y cognitivo) y } \\
\text { articulación entre ellas (co- } \\
\text { ocurrencias). }\end{array}$ \\
\hline & Datos & $\begin{array}{l}\text { Utilización de datos como } \\
\text { argumento. }\end{array}$ \\
\hline \multirow[t]{4}{*}{ El discurso en campaña } & Temas & $\begin{array}{l}\text { Ítems que articulan el texto, } \\
\text { propuestas. }\end{array}$ \\
\hline & Tono & $\begin{array}{l}\text { Sentimiento sugerido en el } \\
\text { discurso (emocional, positivo, } \\
\text { negativo). }\end{array}$ \\
\hline & Representación propia & $\begin{array}{l}\text { Caracterización de sí mismo y del } \\
\text { equipo. }\end{array}$ \\
\hline & Representación del adversario & $\begin{array}{l}\text { Caracterización de } \\
\text { adversarios políticos. }\end{array}$ \\
\hline
\end{tabular}

Fuente: Elaboración propia.

\section{PRINCIPALES HALLAZGOS}

\subsection{LAS CARACTERÍSTICAS NO POPULISTAS DE ARAUZ}

La primera pregunta de investigación plantea si, dado que Arauz es el sucesor designado por Correa y comparten características objetivables (Alcántara et. Al, 2016), también hereda del expresidente los rasgos del discurso populista (De la Torre, 2012; Freidenberg, 2012; Chavero, 2017). Los resultados indican que Arauz no mantiene características populistas, aunque hace algunas referencias al poder popular o a ser la voz de los ciudadanos: «Las bases, el pueblo, las mayorías están con 
\#ElBinomioDeLaEsperanza» (03.02.2021 $\left.{ }^{9}\right)$. Cuando utiliza la categoría pueblo, lo presenta conformado por las clases populares y las minorías tradicionalmente excluidas de la vida política.

Frente a la polarización que presentaba el discurso de Correa (en el que confrontaba permanentemente al pueblo y a las élites), Arauz llama a la unidad de todos los sectores sociales: «No nos motiva el odio, no nos motiva la mala onda ni la bronca entre políticos. Queremos soluciones para nuestro pueblo y lo vamos a lograr con nuestra juventud, innovación y creatividad» (23.02.2021); «Sí se puede lograr la unidad. Vamos adelante con el gran bloque: progresismo + unidad plurinacional + socialdemocracia» (08.02.2021). Siguiendo esta línea de no confrontar, se refiere poco a sus adversarios políticos, aunque en algunas ocasiones se refiere a Guillermo Lasso, para lo cual recurre a la categorización (banquero) y a la comparación, intentando diferenciarse de su adversario: «Y si le das 1.000 millones a los banqueros lo guardan en sus cuentas offshore en Miami. En eso se parecen la economía y el poder: solo funciona cuando se comienza por las mayorías» (22.01.2021).

En su discurso, aunque sí incluye a la patria (en 6 ocasiones) y a los patriotas (9), lo hace para posicionar la idea de recuperar la patria tras los cuatro años del gobierno de Moreno, pero no con la fuerza que adquiría la categoría en el discurso del anterior presidente (Chavero, 2017). Tampoco se identifica en Arauz el recurso a los propietarios de los grandes medios de comunicación, que sí era una constante en el discurso de Correa (Ibid.).

Frente al lenguaje más cercano y directo de Correa, los resultados muestran que en el discurso de Arauz prima el lenguaje correcto, sobrio y técnico, muchas veces sofisticado: «Estas acciones, reñidas con la práctica del periodismo de excelencia y del juego limpio en democracia constituyen, además, una afrenta al buen nombre y a la honra de mi persona y de la de la organización política que lidera la intención de voto en mi país» (29.01.2021). En algunas ocasiones el lenguaje se refina con la incorporación de citas literarias o incluso tuits en inglés y ruso, en una muestra del dominio de varios idiomas del candidato.

A pesar del tono predominante de altura del discurso, en algunos momentos utiliza un lenguaje más cercano: Mil de una, (en referencia a una de las propuestas de campaña y retomando la expresión coloquial ecuatoriana de una); «trujillato» (13.01.2021) (en referencia al desmantelamiento de la institucionalidad liderado por Trujillo como presidente del CPCCS-T), «hay que dar una mano» (17.01.2021) o «alivio de una». En general, el emisor original de los mensajes con este tipo de lenguaje no es la cuenta oficial del candidato, sino que son retuits: «por un pucho de billete se venden» (retuit de @enriquemenoscal, director nacional de Centro Democrático, 02.01.2021) o «no voten por un meme» (retuit de @VerdugaAbraham, comunicador, recogiendo un comentario que Arauz realizó en el debate presidencial, 18.01.2021). Tal y como se observa, los casos en los que Arauz retuitea otros mensajes lo hace con las publicaciones de miembros del mismo movimiento político o actores afines, lo que refleja un ejercicio de autorreferencialidad típico del uso político de Twitter en período electoral (García-Ortega y Zugasti-Azagra, 2018).

\footnotetext{
${ }^{9}$ Las fechas expresadas entre paréntesis se refieren al día de publicación del tuit que contiene la cita mencionada.
} 


\subsection{LA CONSTRUCCIÓN DEL DISCURSO EN REDES}

\subsubsection{Los campos semánticos}

Para identificar cómo Arauz construye su discurso en redes, partimos de una revisión de las palabras más utilizadas, encontrando que la que más emplea es mil (en 88 ocasiones), en referencia a la propuesta de campaña de entregar mil dólares a un millón de mujeres cabezas de familia para superar la crisis agravada por la pandemia. Tras ella, Ecuador (20) y electoral (12) hacen referencia a las dos principales dimensiones del discurso: recuperar Ecuador y alertar sobre las irregularidades del proceso electoral. Otros términos que emplea son trabajo (10), esperanza (9) y futuro (9), con los que fortalece los pilares del discurso de campaña. A partir de aquí, identificamos cuáles son los principales pilares semánticos sobre los cuales se articula el discurso de la primera vuelta del candidato Andrés Arauz. La siguiente ilustración recoge tanto los campos como la manera en que se relacionan.

\section{Ilustración 1. Red de relaciones entre las categorías semánticas del discurso de Arauz ( $1^{\mathrm{a}}$ vuelta elecciones 2021)}

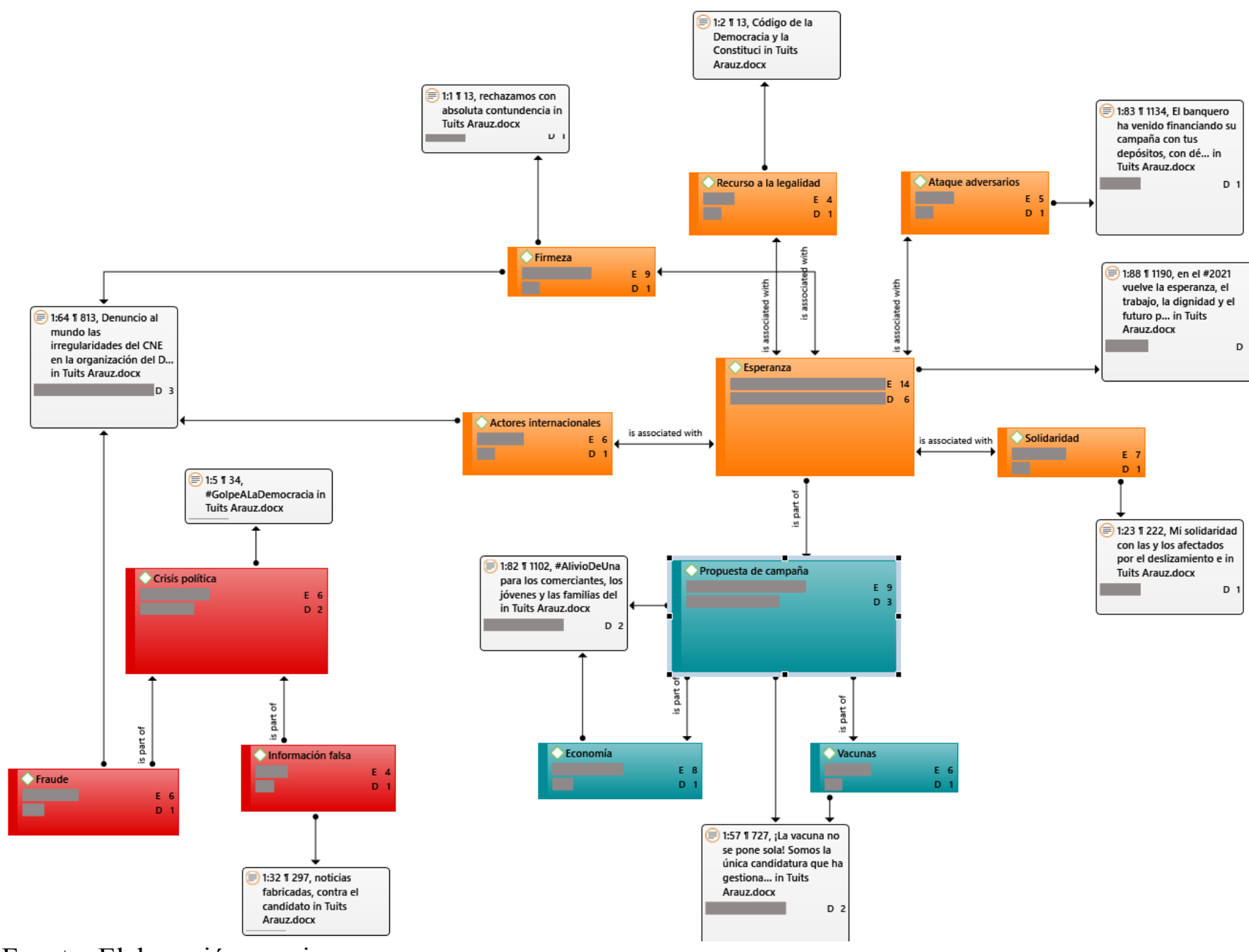

Fuente: Elaboración propia 
Tal y como se observa en la ilustración, el discurso de Arauz gira en torno a tres categorías: esperanza, propuestas de campaña y crisis política. La primera de ellas es la principal, la esperanza, en torno a la que se articula el discurso del candidato en redes (y la campaña) y que coincide con el nombre de su organización política (Unión por la Esperanza, UNES). Este campo (que aparece en 14 ocasiones) está relacionado con otros 6 de los analizados (dato que indica D), a través de los cuales se construye el discurso con los otros pilares temáticos: propuestas de campaña y crisis política.

La construcción del campo esperanza tiene dos dimensiones; la primera de ella es de naturaleza positiva y es la que se relaciona con acontecimientos coyunturales (Solidaridad) y con aquellos en los que se lleva la iniciativa y se intentan posicionar los mensajes principales (Propuestas de campaña). La segunda dimensión tiene un tono negativo y se asocia tanto a la caracterización del candidato frente al resto de candidaturas (Ataque al adversario) como al desarrollo del proceso electoral y las denuncias de irregularidades (Firmeza; Recursos a la legalidad y Actores internacionales). De esta manera, el discurso del candidato prioriza las propuestas de campaña con un tono positivo desde el enfoque de la esperanza, pero no renuncia a construir su imagen como un líder distinto mediante el énfasis en las debilidades de sus adversarios (sobre todo del otro candidato en segunda vuelta, Lasso).

La asociación entre la esperanza y las propuestas de campaña es el eje vertebrador del discurso político en redes de Arauz, ya que constituye la principal apuesta del candidato presidencial. En este sentido, las propuestas de campaña que más peso tuvieron en Twitter fueron economía y vacunas (covid-19). Frente a esta construcción del discurso, de tono positivo y que responde a la estrategia de la campaña del candidato, encontramos la asociación con el tercer campo semántico, la crisis política. El protagonismo de este tercer campo (que se observa en 6 ocasiones) responde al desarrollo del proceso electoral y a las denuncias de irregularidades realizadas por varios candidatos. Este campo está construido por tuits categorizados como fraude e información falsa, en respuesta al intento de violentar el proceso electoral (fraude) y a las acusaciones de financiación ilícita que circularon durante la última semana de campaña (información falsa). Esto es, a partir de la denuncia de fraude y de información falsa se construye la propuesta de una crisis política durante la campaña electoral en Ecuador. Es importante destacar que la cuenta oficial de Arauz menciona en escasas ocasiones la palabra fraude y, en su lugar, habla de golpe a la democracia. La utilización de estos conceptos indica que la preocupación principal no tiene que ver con una perspectiva individual o partidaria (ganar o perder la contienda electoral), sino con una de naturaleza general (respetar o no el proceso democrático) y, por tanto, con la preocupación por regenerar la vida democrática (H2).

\subsubsection{Construcción lingüística del discurso de Arauz}

Además de los campos semánticos, identificamos cómo se relacionan las distintas categorías reconocidas en la construcción del discurso del candidato. La siguiente tabla muestra las principales co-ocurrencias halladas en el discurso de Arauz en redes; esto indica la presencia de dos o más códigos en la misma cita, es decir, la presencia de distintas variables de análisis en el mismo tuit. Asimismo, indica los coeficientes que representan esas co-ocurrencias, lo que informa sobre la intensidad en esas relaciones. 
Tabla 2. Co-ocurrencias (y coeficientes) entre las categorías de análisis

\begin{tabular}{|c|c|c|c|c|c|c|c|c|c|c|c|c|}
\hline & $\begin{array}{l}\text { Act } \\
\text { ore } \\
\text { s } \\
\text { inte } \\
\text { rna } \\
\text { cio } \\
\text { nal } \\
\text { es }\end{array}$ & $\begin{array}{l}\mathbf{A} \\
\mathbf{g} \\
\mathbf{u} \\
\mathbf{a}\end{array}$ & $\begin{array}{l}\text { Ata } \\
\text { que } \\
\text { adve } \\
\text { rsari } \\
\text { os }\end{array}$ & $\begin{array}{l}\text { cor } \\
\text { rup } \\
\text { ció } \\
\text { n }\end{array}$ & $\begin{array}{l}\text { Ec } \\
\text { on } \\
\text { o } \\
\text { mí } \\
\text { a }\end{array}$ & $\begin{array}{l}\text { Em } \\
\text { ple } \\
\text { o }\end{array}$ & $\begin{array}{l}\mathbf{F i} \\
\mathbf{r m} \\
\mathrm{ez} \\
\mathrm{a}\end{array}$ & $\begin{array}{l}\text { F } \\
\mathbf{r} \\
\mathbf{a} \\
\mathbf{u} \\
\mathbf{d} \\
\mathrm{e}\end{array}$ & $\begin{array}{l}\text { M } \\
\text { uj } \\
\text { er } \\
\text { es }\end{array}$ & $\begin{array}{l}\text { Pro } \\
\text { pue } \\
\text { sta } \\
\text { de } \\
\text { cam } \\
\text { pañ } \\
\text { a }\end{array}$ & $\begin{array}{l}\text { Soli } \\
\text { dar } \\
\text { ida } \\
\text { d }\end{array}$ & $\begin{array}{l}\text { Va } \\
\text { cu } \\
\text { na } \\
\text { s }\end{array}$ \\
\hline $\begin{array}{l}\text { Actores } \\
\text { internacionales }\end{array}$ & 0 & 0 & 0 & 0 & 0 & 0 & $\begin{array}{l}1 \\
(0,07 \\
)\end{array}$ & $\begin{array}{l}1 \\
(0,0 \\
9)\end{array}$ & 0 & 0 & $\begin{array}{c}1 \\
(0,08)\end{array}$ & 0 \\
\hline Agua & 0 & 0 & 0 & 0 & 0 & 0 & 0 & 0 & 0 & $\begin{array}{r}1 \\
(0,11)\end{array}$ & 0 & 0 \\
\hline $\begin{array}{l}\text { Ataque } \\
\text { adversarios }\end{array}$ & 0 & 0 & 0 & 0 & $\begin{array}{l}1 \\
(0,0 \\
8)\end{array}$ & 0 & 0 & 0 & 0 & 0 & 0 & 0 \\
\hline Corrupción & 0 & 0 & 0 & 0 & 0 & 0 & $\begin{array}{l}1 \\
(0,10 \\
)\end{array}$ & 0 & 0 & 0 & 0 & $\begin{array}{r}1 \\
(0,14 \\
)\end{array}$ \\
\hline Economía & 0 & 0 & $\begin{array}{r}1 \\
(0,08)\end{array}$ & 0 & 0 & 0 & 0 & 0 & 0 & $\begin{array}{r}3 \\
(0,21)\end{array}$ & 0 & 0 \\
\hline Empleo & 0 & 0 & 0 & 0 & 0 & 0 & 0 & 0 & 0 & $\begin{array}{r}1 \\
(0,11)\end{array}$ & 0 & 0 \\
\hline Firmeza & $\begin{array}{r}1 \\
(0,07)\end{array}$ & 0 & 0 & $\begin{array}{r}1 \\
(0,10)\end{array}$ & 0 & 0 & 0 & $\begin{array}{r}1 \\
(0,0 \\
7)\end{array}$ & 0 & 0 & 0 & 0 \\
\hline Fraude & $\begin{array}{r}1 \\
(0,09)\end{array}$ & 0 & 0 & 0 & 0 & 0 & $\begin{array}{r}1 \\
(0,07 \\
)\end{array}$ & 0 & 0 & 0 & 0 & 0 \\
\hline Mujeres & 0 & 0 & 0 & 0 & 0 & 0 & 0 & 0 & 0 & $\begin{array}{r}1 \\
(0,11)\end{array}$ & 0 & 0 \\
\hline $\begin{array}{l}\text { Propuesta de } \\
\text { campaña }\end{array}$ & 0 & $\begin{array}{r}1 \\
(0, \\
11) \\
\end{array}$ & 0 & 0 & $\begin{array}{r}3 \\
(0,2 \\
1) \\
\end{array}$ & $\begin{array}{r}1 \\
(0,11)\end{array}$ & 0 & 0 & $\begin{array}{r}1 \\
(0,1 \\
1) \\
\end{array}$ & 0 & 0 & $\begin{array}{r}3 \\
(0,25\end{array}$ \\
\hline Solidaridad & $\begin{array}{r}1 \\
(0,08) \\
\end{array}$ & 0 & 0 & 0 & 0 & 0 & 0 & 0 & 0 & 0 & 0 & 0 \\
\hline Vacunas & 0 & 0 & 0 & $\begin{array}{r}1 \\
(0,14)\end{array}$ & 0 & 0 & 0 & 0 & 0 & $\begin{array}{r}3 \\
(0,25)\end{array}$ & 0 & 0 \\
\hline
\end{tabular}

Fuente: Elaboración propia.

La tabla indica que la mayor co-ocurrencia en las propuestas de campaña es con economía y vacunas; es decir, las citas en las que se realizan propuestas de campaña corresponden a las propuestas relativas a la reactivación de la economía y a la adquisición 
de vacunas contra el covid-19, así como el plan de vacunación, tal y como ilustran los siguientes ejemplos.

\section{Ilustración 2. Temas de campaña posicionados por Arauz en Twitter}
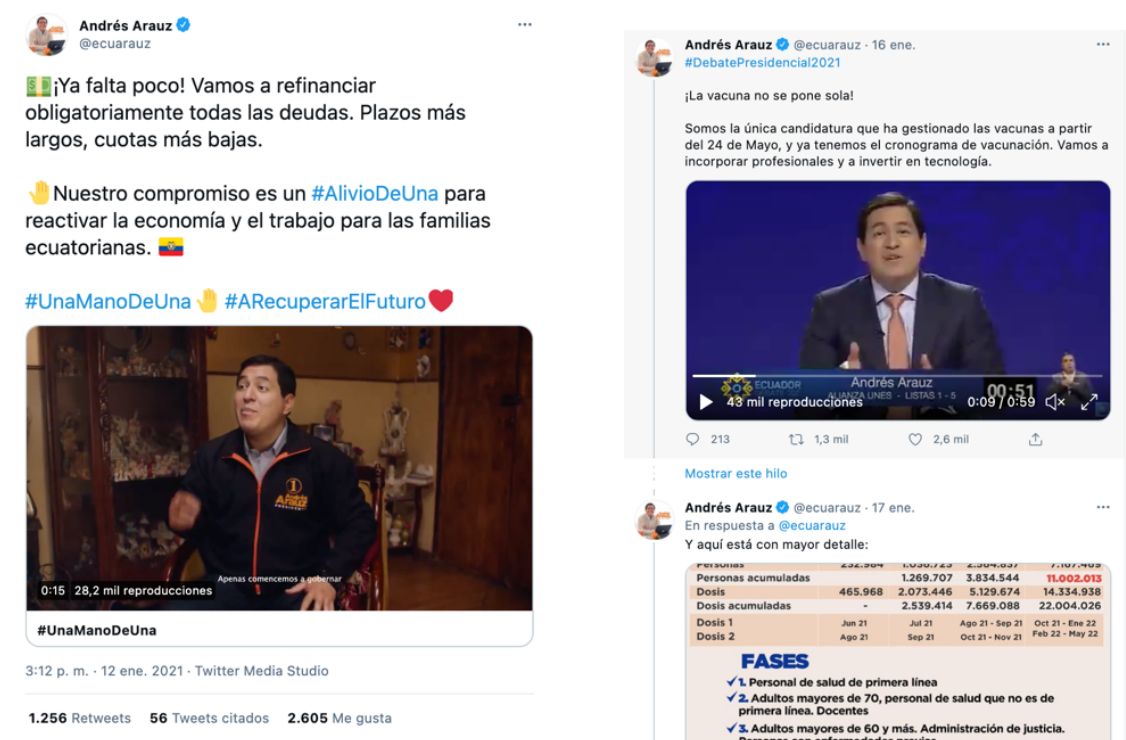

Fuente: https://twitter.com/ecuarauz/status/1349086776921612289 https://twitter.com/ecuarauz/status/1350654794789216257

En segundo lugar, destaca la relación entre el fraude, la firmeza y los actores internacionales, es decir, estos recursos se utilizan en la misma frase o tuit. Esta relación responde a las denuncias del candidato de irregularidades en el proceso y la firmeza frente a esta situación, en la que pide a los actores internacionales (UE, OEA y observadores electorales internacionales) vigilar el proceso.

\section{Ilustración 3. Denuncia del candidato Arauz ante irregularidades del proceso electoral}

Andrés Arauz

@ecuarauz

Denuncio al mundo las irregularidades del CNE en la organización del Debate Presidencial, prueba de la poca responsabilidad para afrontar con eficiencia un evento central del proceso electoral

Aquí nuestra postura y reclamo:

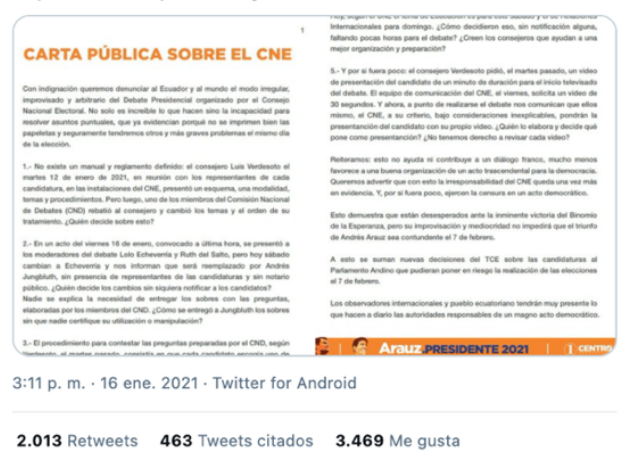

Fuente: https://twitter.com/ecuarauz/status/1350536180803248129 
Los coefecientes de co-ocurrencias indican una baja intensidad (no superior a 0,3 ), lo cual refleja que en general los mensajes de Arauz tratan de ser claros, con una sola información en cada uno de los tuits y argumentándola en cada uno de los casos, pero sin relacionarla con otros mensajes o temas de la campaña. De esta manera, cada tuit se enfoca en un mensaje.

Uno de los elementos que destaca en la tabla es la ausencia de co-ocurrencias entre la esperanza y el resto de campos. Esto responde a que los mensajes categorizados como esperanza se centran en insuflar esa emoción en los simpatizantes y posibles votantes de UNES, sin incluir ningún otro mensaje; la intención de los tuits de esperanza, por tanto, no es transmitir mensajes con propuestas específicas de campaña o temas de debate público, sino recuperar la esperanza para superar la crisis económica, sanitaria y política. Esto se refuerza con el empleo de un lenguaje que invita a la acción: A recuperar el futuro.

\section{Ilustración 4. Mensaje de esperanza de Arauz durante la campaña electoral ecuatoriana}

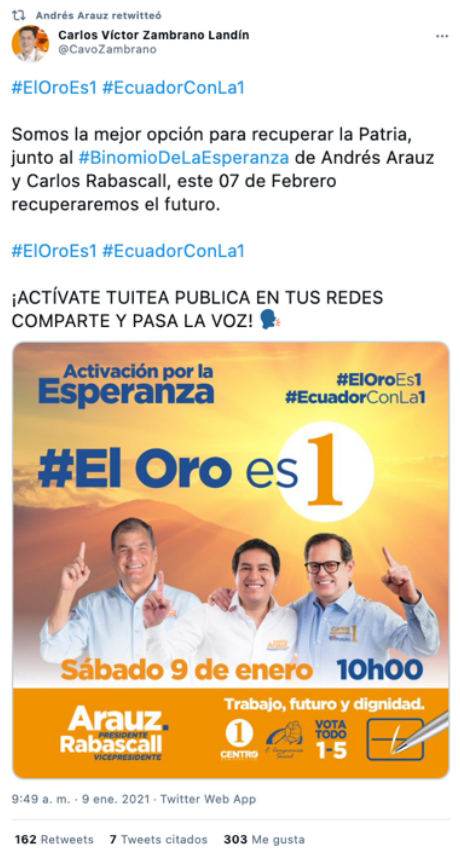

Fuente: https://twitter.com/ecuarauz/status/1347924390063824898

\subsection{LOS RASGOS ELECTORALES}

La tercera pregunta de investigación proponía identificar si las características subjetivables (Alcántara et al., 2016) de Arauz se reflejan en el discurso de campaña y cómo este se impregna de rasgos electorales. Los resultados indican que Arauz recurre a herramientas normativas y apela a organismos internacionales: «Pedimos a los Observadores Internacionales que soliciten al CNE la aplicación de la Guía para elecciones en tiempos de pandemia y que cumplan con los estándares de Open Data de las actas electorales en tiempo real» (13.01.2021); "Solicito al CNE que de acuerdo al criterio vinculante del Procurador y al derecho constitucional, permita que se respete el derecho constitucional a votar a quienes estén en la fila a las 17h00» (07.02.2021). En algunas ocasiones también incorpora cifras: «Bolivia: 92.000 cuentas falsas para apoyar el Golpe de Estado a @evoespueblo. Ecuador: 258.000 cuentas falsas para apoyar a Lasso 
$\mathrm{y}$ violentarnos a nosotros, inventar y viralizar falsas noticias» (04.02.2021). De esta forma, el candidato intenta dotar su discurso de autoridad y fortalecerlo.

El tono elevado y sofisticado («Rechazamos con absoluta contundencia la interferencia y la grosera intromisión de otras funciones del Estado en la función electoral»(21.02.2021)), coherente con sus características subjetivables como líder, convive con un intento por destacar la cercanía del candidato con el ciudadano medio y humanizarlo: «Ayer mi mamá me sorprendió durante la caminata en Quito» (28.01.2021); «En 2015 me comprometí, por mi hijo» (18.01.2021); «Hoy vivimos la fiesta democrática, yo lo hago como siempre, acompañando a mi abuelita» (07.02.2021), para lo cual también se apela al uso de emoticonos como parte del lenguaje de redes sociales. De esta manera, Arauz se identifica con el ciudadano medio como una herramienta de acercamiento en período electoral: «He sido migrante, sé lo que es estar lejos de la familia, sacrificarse por el futuro y añorar la Patria» (16.02.2021); «Sabemos que la mejor forma de cuidar de las familias es con un gobierno que entienda sus necesidades» (31.12.2021). Esa representación de sí mismo como alguien cercano convive con el énfasis en las capacidades propias del candidato y de su equipo, lo cual se refleja en otras construcciones además de la utilización de varios idiomas ya citada: «Nosotros sabemos cómo hacerlo» (18.01.2021); «Tenemos la experiencia y la capacidad para acompañar a las familias golpeadas por la crisis sanitaria y económica» (15.01.2021). Así, recurre a la autopresentación positiva como una macroestrategia semántica (Van Dijk, 2005) típica de las campañas electorales y que se diferencia de la propuesta del líder redentor que se sacrifica a sí mismo característico de los discursos populistas.

En esa misma línea, sus tuits apuestan por la colaboración, invitando a participar de la campaña y del proyecto político: «Pasa la voz» (09.01.2021), «Súmate a la revolución de los medios digitales» (14.01.2021), «Únete a la campaña del cambio, la alegría y la prosperidad» (21.01.2021); «Construyamos juntos un conocimiento soberano para nuestra América» (12.01.2021).

En términos de replicabilidad del mensaje (Cotarelo, 2012), encontramos una amplia diferencia entre los distintos mensajes. En primer lugar, destaca la alta difusión del tuit en el que anuncia los resultados electorales (12.128 RT), muy por encima del resto de mensajes que más se replicaron durante la campaña. Dentro de ellos, los que más se difundieron fueron aquellos en los que se defiende de las acusaciones falsas (5.455 RT), la situación sanitaria (3.368 RT) y las alertas ante una posible violación del proceso electoral (3.242 RT).

\section{A MODO DE CONCLUSIÓN}

Este trabajo se proponía analizar las características del discurso en Twitter de Andrés Arauz como candidato a la presidencia de la República de Ecuador durante la primera vuelta de las elecciones de 2021. Para ello, partimos de identificar si su discurso repetía rasgos populistas de su mentor y anterior presidente, Rafael Correa. El análisis realizado da cuenta de que, aunque comparten algunas características como líderes políticos, el discurso de Arauz no tiene rasgos populistas, salvo la utilización de la categoría pueblo que utiliza para expresar el apoyo de las bases y mínimamente ataca a sus adversarios, lo que confirma solo parcialmente la $\mathrm{H} 1$.

En segundo lugar, nos proponíamos identificar cuáles son los principales campos semánticos y las particularidades de su discurso en redes sociales. El análisis muestra que el discurso durante la primera vuelta se articuló en torno a tres grandes campos: la esperanza (eje principal del discurso y de la campaña), las propuestas de campaña (sobre todo las relacionadas con el empleo y el plan de vacunación) y la crisis política (construida 
a partir de irregularidades en el proceso electoral y de la propagación de noticias falsas contra el candidato Arauz), respondiendo así a la H2. Estos hallazgos son coincidentes con lo que propone la literatura académica con respecto a los temas utilizados por los líderes populistas de izquierda: problemas sociales y regeneración democrática (AlonsoMuñoz y Casero-Ripollés, 2018), a pesar de que Arauz no tiene un discurso populista.

En tercer lugar, queríamos conocer cómo las características subjetivables se reflejan en el tipo de lenguaje que Arauz emplea en redes sociales. En este sentido, encontramos que utiliza un lenguaje bastante técnico, en el que incluye referencias a normativas y llama a la unidad frente a la polarización de los discursos populistas (De la Torre, 2012; Chavero, 2017; Pérez-Curiel, Rivas de Roca y García-Gordillo, 2021) y a la participación de los ciudadanos, pero también muestra firmeza frente a posibles irregularidades en el proceso y se articula con sus semejantes internacionales. El tono del lenguaje de Arauz es elevado y sofisticado pero al mismo tiempo realiza algunos intentos de humanizar al candidato y presentarse como un ciudadano más, confirmando lo propuesto por H3. Esta inclusión de información personal es coincidente con análisis previos de otros líderes políticos (Pérez Curiel, 2020).

Los hallazgos encontrados indican que el discurso del candidato correísta Andrés Arauz tiene características propias, que lo alejan del estilo discursivo que caracterizó las alocuciones del expresidente y sugieren una nueva etapa en el progresismo ecuatoriano. En este trabajo nos hemos centrado en el análisis del discurso en redes sociales digitales, en estudios posteriores indagaremos en el discurso del candidato en otros espacios y en otros momentos no electorales, lo que nos permitirá profundizar en las características y estilo discursivo de los nuevos líderes políticos de América Latina.

\section{REFERENCIAS BIBLIOGRÁFICAS}

Alcántara, Manuel, Barragán, Mélany y Sánchez, Francisco. 2016. «Los presidentes latinoamericanos y las características de la democracia». Colombia Internacional, 87: 21-52.

Alonso-Muñoz, Laura y Casero-Ripollés, Andreu. 2018. «Communication of European populist leaders on Twitter: Agenda setting and the 'more is less' effect». El profesional de la información, 27 (6): 1193-1202.

Arias, Andrés y Chiriboga, Leonardo. 2020. «La ruta al "paquetazo" y el retorno de la economía fondomonetarista al Ecuador». En Ramírez, Franklin (ed). Octubre y el derecho a la resistencia. Revuelta popular y neoliberalismo autoritario en Ecuador. (pp.169-193). Buenos Aires: CLACSO.

Bouza, Fermín. 2007. «Populismo y Medios de Comunicación». Ponencia presentada en el seminario Populismo. Centro de Cultura Contemporánea de Barcelona. Barcelona (España), 16-17 de abril de 2007.

Calvo, Ernesto y Aruguete, Natalia. 2020. Fake news, trolls y otros encantos. Buenos Aires: Siglo XXI Editores.

Canel, María José. 1998. «Los efectos de las campañas electorales». Comunicación y Sociedad, 11(1): 47-67.

Cárdenas, Alejandro, Ballesteros, Carlos y Jara, René. 2017. «Redes sociales y campañas electorales en Iberoamérica. Un análisis comparativo de los casos de España, México y Chile». Cuadernos.info, 41: 19-40. 
Carsten Reinemann, Toril Aalberg, Frank Esser, Jesper Strömbäck y Claes H. de Vreese. 2016. «Populist Political Communication Toward a Model of Its Causes, Forms, and Effects». En Populist Political Communication in Europe, ed. Toril Aalberg, Frank Esser, Carsten Reinemann, Jesper Strömbäck, Claes H. de Vreese (pp.1225). New York/London: Routledge.

Cotarelo, Ramón. 2012. «La expansión de la ciberpolítica». En España en crisis, eds. Colino, C. y Cotarelo, R. (pp. 331-357). Valencia: Tirant lo Blanch.

Charaudeau, Patrick. 2009. «Análisis del discurso e interdisciplinariedad en las ciencias humanas y sociales». El discurso y sus espejos. México: Universidad Nacional Autónoma de México.

Chavero, Palmira. 2013. «Medios de comunicación y redes sociales en las democracias actuales: ¿Complementariedad o sustitución? Una aproximación al caso español». En Ciberpolítica: las nuevas formas de acción y movilización política, comp. Cotarelo, R. Valencia: Tirant lo Blanch.

Chavero, Palmira. 2017. «Populismo en el Discurso Político y Mediático en América Latina: El Caso de Rafael Correa en Ecuador». I-Land Journal: identity, Language and Diversity (2): 102-120.

D’Alessandro, Martín. 2004. «¿Qué es la personalización de la política? Algunos hallazgos en los medios gráficos, 1983-1995». Revista Argentina de Ciencia Política, 7-8: 73-94.

D'Alessandro, Martín. 2006. «Liderazgo político». En Política. Cuestiones y problemas, comp. Luis Aznar y Miguel De Luca (pp. 305-336). Buenos Aires: Ariel.

De la Torre, Carlos. 2012. «Rafael Correa, un Populista del Siglo XXI». En ¿Qué Democracia en América Latina?, ed. Cheresky, Isidoro (pp. 251-281). Buenos Aires: CLACSO/Prometeo.

De la Torre, Carlos. 2013. «El Populismo Latinoamericano: Entre la Democratización y el Autoritarismo». Nueva Sociedad, 247: 120-137.

De Vreese, Claes. 2004. "The effects of frames in political television news on issue interpretation and frame salience». Journalism \& Mass Communication Quarterly, 81 (1): 36-52.

Deiwiks, Christa. 2009. «Populism». Living Reviews in Democracy, 1: 1-9.

Fairclough, Norman. 1995. Critical discourse analysis. The critical study of language. London and New York: Longman.

Freidenberg, Flavia. 2012. «El Flautista de Hammelin: liderazgo y populismo en la democracia ecuatoriana». En El retorno del pueblo, eds. Peruzzotti, E. y De la Torre, C. pp. (185-233). Quito: FLACSO Ecuador.

Freidenberg, Flavia y Pachano, Simón. 2016. El sistema político ecuatoriano. Quito: FLACSO.

García Beaudoux, Virginia y D’Adamo, Orlando. 2004. «Campañas electorales y sus efectos sobre el voto. Análisis de la campaña electoral presidencial 2003 en Argentina». Psicología Política, 28: 7-25.

García-Ortega, Carmela y Zugasti-Azagra, Ricardo. 2018. «Gestión de la campaña de las elecciones generales de 2016 en las cuentas de Twitter de los candidatos: entre la 
autorreferencialidad y la hibridación mediática». El profesional de la información, 27 (6): 1215-1224.

Gitlin, Todd. 1991. «Blips, bytes and savvy talk: television's impact on American Politics». En Communication and citizenship, eds. Dahlgren, P. y Sparks, C. (pp.119-136). Boston: Routledge.

Guamán, Adoración. 2020. «Fin de Estado de derecho y la protesta popular». En Octubre y el derecho a la resistencia. Revuelta popular y neoliberalismo autoritario en Ecuador, ed. Ramírez (pp. 149-168). F. Buenos Aires: CLACSO.

Hermet, Guy. 2003. «El Populismo como Concepto». Revista de Ciencia Política 23/1: $5-18$.

Jagers, Jan, y Walgrave, Stefaan. 2007. «Populism as political communication style: An empirical study of political parties' discourse in Belgium». European Journal of Political Research, 46(3): 319-345.

Laclau, Ernesto. 2005a. La Razón Populista. Buenos Aires: Fondo de Cultura Económica.

Laclau, Ernesto. 2005b. «La Deriva Populista y la Centroizquierda Latinoamericana». Nueva Sociedad, 205: 56-61.

López-Meri, Amparo, Marcos-García, Silvia y Casero-Ripollés, Andreu. 2017. «What do politicians do on Twitter? Functions and communication strategies in the Spanish electoral campaign of 2016». El profesional de la información, 26 (5): 795-804.

López-Rabadán, Pablo, López-Meri, Amparo y Doménech-Fabregat, Hugo. 2016. «La imagen política en Twitter. Usos y estrategias de los partidos políticos españoles». index.comunicación, 6 (1): 165-195.

Martínez y Coma, Ferrán. 2008. «¿Cuáles fueron los efectos de la campaña electoral española del 2000?». REIS, 112/05: 231-257.

Mazzoleni, Gianpetro y Schulz, Winfried. 1999. «Mediatization of Politics: A Challenge for Democracy?». Political Communication, 16 (3): 247-261.

Muñoz Alonso, Alejandro y Rospir, Juan Ignacio. 1995. Comunicación Política. Madrid: Ed. Universitas.

Ortega, Félix. 2011. La Politica Mediatizada. Madrid: Alianza.

Paletz, David. 1997. «Campañas y elecciones». CIC, 3: 205-227.

Papacharissi, Zizi. 2002. "The virtual sphere». New media and society, 4 (1): 9-27.

Pérez-Curiel, Concha, Rivas-de-Roca, Rubén y García-Gordillo, Mar. 2021. «Impact of Trump's Digital Rhetoric on the US Elections: A View from Worldwide Far-Right Populism». Social Sciences 10: 152.

Pérez-Curiel, Concha. 2020. «Trend towards extreme right-wing populism on Twitter. An analysis of the influence on leaders, media and users». Communication \& Society, 33: 175-92.

Petracca, Orazio. 1997. «Liderazgo». En Diccionario de política, dirs. Bobbio, Norberto, Matteucci, Nicola y Pasquino, Gianfranco, (pp.914-918) México D.F.: Siglo XXI. [Dizionario di politica, 1976.]

Vallés, Josep María. 2010. «Política democrática y comunicación: un rapto consentido». Revista de Estudios Politicos, 150: 11-50.

Van Dijk, Teun. 2009. Discurso y Poder. Barcelona: Editorial Gedisa. 
Van Dijk, Teun. 2005. «Política, ideología y discurso». QUÓRUM ACADÉMICO, 2 (2): 15-47.

Van Dijk, Teun 1999. «El Análisis Crítico del Discurso». Anthropos, 186: 23-36.

Yang, Xinxing., Chen, Bo-Chiuan., Maity, Mrinmoy y Ferrara, Emilio. 2016. «Social politics: agenda setting and political communication on social media». En International conference on social informatics. Eds. Spiro, E. y Ahn, (pp. 330344). Y. Bellevue, WA: Springer. Springer. 